Research Paper

\title{
Exploration of the optimal treatment regimes for Esthesioneuroblastoma: a single center experience in China
}

\author{
Yujie Yuan ${ }^{*}$, Jinning Ye ${ }^{2 *}$, Huizhi Qiu ${ }^{3 *}$, Shaoqing Niu${ }^{1}$, Bixiu Wen², Dongping Wang ${ }^{\natural 凶}$, Xinping Cao ${ }^{5 凶}$ \\ and Yufeng Ren ${ }^{1 \times}$ \\ 1. Department of Radiation Oncology, The First Affiliated Hospital, Sun Yat-sen University; \\ 2. Center of Gastrointestinal Surgery, The First Affiliated Hospital, Sun Yat-sen University; \\ 3. Department of Radiotherapy, cancer center of Guangzhou medical university, Guangzhou 510080, P.R. China; \\ 4. Department of Organ transplantation, The First Affiliated Hospital, Sun Yat-sen University; \\ 5. Department of Radiation Oncology, Cancer Center of Sun Yat-sen University, State Key Laboratory of Oncology in South China, Collaborative Innovation \\ Center for Cancer Medicine. \\ * These authors contributed equally to this work.
}

$\triangle$ Corresponding authors: Yufeng Ren., M.D., Ph.D, Department of Radiation Oncology, The First Affiliated Hospital, Sun Yat-sen University, Guangzhou 510060, P.R. China. E-mail: renyuf@mail.sysu.edu.cn

(c) Ivyspring International Publisher. This is an open access article distributed under the terms of the Creative Commons Attribution (CC BY-NC) license (https://creativecommons.org/licenses/by-nc/4.0/). See http://ivyspring.com/terms for full terms and conditions.

Received: 2017.06.23; Accepted: 2017.10.15; Published: 2018.01.01

\begin{abstract}
BACKGROUND: Esthesioneuroblastoma (ENB) is an uncommon neoplasm arising from the olfactory mucosa. The optimal treatment regimen for ENB remains unclear. This study aims to evaluate its clinical features, long-term outcomes and explore optimal treatment patterns.

METHODS: Clinical data of consecutive 44 ENB patients were reviewed retrospectively. The correlation between clinical features and treatment approaches were analyzed, with several prognostic factors explored meanwhile.

RESULTS: The age of onset of ENB showed a bimodal distribution, with peaks at 10 20 and 50 60 years. The median follow-up time was 84 months (range, 27 198 months). The 5-year overall and progression free survival rates were $42.7 \%$ and $39.1 \%$, respectively, with 10 -year rates of $28.9 \%$ and $21.7 \%$ respectively. Overall, 19 patients developed recurrent disease. Patients undergoing surgery combined with adjuvant radiotherapy had significantly higher 5 -year overall survival $(67.5 \%$ vs. $33.3 \%, P=0.043)$ and progress-free survival $(60.0 \%$ vs. $18.7 \%, P=0.008)$ than those receiving other treatment approaches. No-Skin-involved ENB was associated with markedly better 5 -year overall survival $(45.5 \%$ vs. $0 \%, \mathrm{P}=0.038)$ and progress-free survival $(31.3 \%$ vs. $0 \%, \mathrm{P}=0.001)$ compared with skin-involved tumor.

CONCLUSIONS: ENB is a rarely malignant tumor with high probability of locoregional recurrence and poor survival. Surgical resection followed by radiotherapy has been shown to achieve optimal local control and overall survival.
\end{abstract}

Key words: Esthesioneuroblastoma; Multimodality; Surgery; Radiotherapy; Survival.

\section{Introduction}

Esthesioneuroblastoma (ENB), also known as olfactory neuroblastoma, is a rare tumor comprising $5.0 \% \sim 13.2 \%$ of malignant neoplasm of the nasal cavity with a prevalence of $0.4 /$ million $^{1-3}$. It has been generally recognized that ENB originates from primitive basal cells of the olfactory epithelium, without available known environmental exposures associated with this neoplasm ${ }^{4-6}$.

Patients with ENB may be treated with various modalities including surgery, radiotherapy (RT), and 
chemotherapy or their combinations ${ }^{7-11}$. Therapeutic strategy may vary significantly among different institutions. Specifically, RT has been used as a preoperative, postoperative, or definitive treatment modality ${ }^{12-14}$. Modern surgical techniques have been shown to dramatically improve disease-free survival ${ }^{15,16}$. Because of low incidence and paucity of reported clinical data, there is still no definitive consensus regarding the optimal treatment forth unusual neoplasm.

The purpose of current study is to explore clinical characteristics of patients with ENB, and to evaluate treatment efficacies of different modalities and prognostic factors. Importantly, an optimal treatment approach for this rare cancer should be addressed by this study.

\section{Materials and Methods}

\section{Patient data}

The medical records of consecutive 54 patients with pathological diagnosis of ENB from July 1986 to February 2011were retrospectively reviewed, the median follow-up for this cohort was 84 months (range, 27 198 months). The protocol of this retrospective study was performed in accordance with the Declaration of Helsinki and was approved by the ethics committee of our hospital. All patients, with30 male and 24 female, were first enrolled in data filtration and analysis (the grading systems at presentation was not evident from the records); whereas one patient with an inadequate treatment record and other nine patients with no treatment were excluded from the final analysis. In sum, Clinical data, including demographics, primary diagnosis, tumor staging, treatment modalities and long-term follow-up outcomes, were collected from 44 patients (Table 1).

\section{Tumor staging}

All patients were staged with head-neck computed tomography (CT) initially and magnetic resonance imaging (MRI) additionally in recent years. The diagnosis suspected on CT and/or MRI was pathologically proven with biopsy. Both Kadish/Morita staging system and American Joint Committee on Cancer (AJCC) stage (2002) were recorded. Of note, nine patients presented with cervical lymph node (LN) metastases ipsilaterally, with other three patients bilaterally involved. Four patients, who had local skin involvement was classified as stage D according to the Kadish/Morita stage system (Fig. 1). MRI and/or CT scans were routinely applied to assess tumor response to chemotherapeutic agents.
Table 1. Characteristics and Treatment modalities for patients with esthesioneuroblastoma

\begin{tabular}{ll}
\hline Characteristics & Value (\%) \\
\hline Total patients & 44 \\
Age (years) & \\
Median at diagnosis & 54 \\
Range & $6-74$ \\
Gender & $23(52.3 \%)$ \\
Male & $21(47.7 \%)$ \\
Female & \\
KPS & $14(31.8 \%)$ \\
90-100 & $30(68.2 \%)$ \\
$80-90$ & \\
Family history & $5(11.4 \%)$ \\
yes & $39(88.6 \%)$ \\
No & \\
Staging & \\
Kadish/Morita stage & $3(6.8 \%)$ \\
A & $16(36.4 \%)$ \\
B & $12(27.3 \%)$ \\
C & $13(29.5 \%)$ \\
D & \\
T classification & $6(13.6 \%)$ \\
T1 & $15(34.1 \%)$ \\
T2 & $13(29.5 \%)$ \\
T3 & $10(22.7 \%)$ \\
T4 & \\
N classification & $35(79.5 \%)$ \\
N0 & $9(20.5 \%)$ \\
N+ & \\
Treatment modalities & \\
Surgery & \\
No surgery & \\
Open craniofacial resection & $9(21(47.7 \%)$ \\
Endoscopic -assisted craniofacial & $5(11.4 \%)$ \\
resection & \\
Endoscopic -definitive surgery & $8(18.1 \%)$ \\
Endoscopic -not definitive surgery & $2(4.5 \%)$ \\
Radiatherapy & \\
Pre-op RT & 0 \\
Post-op RT (50-66Gy) & $15(34.1 \%)$ \\
RT alone (62-76Gy) & \\
Chemo-RT (62-70Gy) & \\
Chemotherapy & \\
Alone & \\
Pre-RT/ surgery & \\
Post-RT/ surgery & \\
Concurrent with RT & \\
\hline Abbreviation. RT: Radiation therapy; KPS: Karnofsky performance status \\
\end{tabular}

\section{Treatment modalities}

To our best knowledge, there is still no definitive consensus regarding the optimal treatment of this unusual neoplasm. Hence, the management of ENB in our unit is also controversial and variable. Patients with confirmed ENB diagnosis were treated with surgical resection, RT and chemotherapy or their combinations. As for surgical procedures, open, endoscopic-assisted craniofacial resection and total endoscopic-definitive resection were performed for ENB patients indicated with surgery. The technique of RT included 2D, 3D and intensity modulated radiation, The techniques of thermoplastic mask, low melting-point lead block, multi-leaf collimator (MLC) 
and source axis distance (SAD) were applied to radiotherapy. 6-8 MV supervoltage $X$ rays generated by a linear accelerator were used for external irradiation. During the two-dimensional conformal radiotherapy, conventional fraction was given, and the dose for the primary lesion was $64 \sim 72 \mathrm{~Gy} / 32 \sim$ $36 \mathrm{~F}$.. supervoltage $X$ rays added beta-rays were used to compensate the dose in consideration of skin and subcutaneous tissues in the neck. The prescribed radiation dosage of 3D-CRT were defined as follows: GTVnx (gross tumor volume): 65-70 Gy; GTVnd (positive lymph nodes volume): 60-70 Gy; CTV60 (clinical target volume 60): 60 Gy; CTVnx50 (clinical target volume 50): 50 Gy; CTVnd50 (nodal clinical target volume): 50 Gy. The prescribed radiation dosage of IMRT was defined as follows: 68 Gy in 30 fractions to the planning target volume (PTV) of the primary gross tumor volume (GTV-P), 60 to 64 Gy to the PTV of nodal gross tumor volume (GTV-N), 60 Gy to the PTV of CTV-1, and 54 Gy to the PTV of CTV-2 and CTV-N. The application of chemotherapy contained huge diversity, with several qualified regimens employed (CAV, EP, MAID, etc.). Tumor response to therapy was routinely assessed by $\mathrm{CT}$ or MRI and ENT examination.

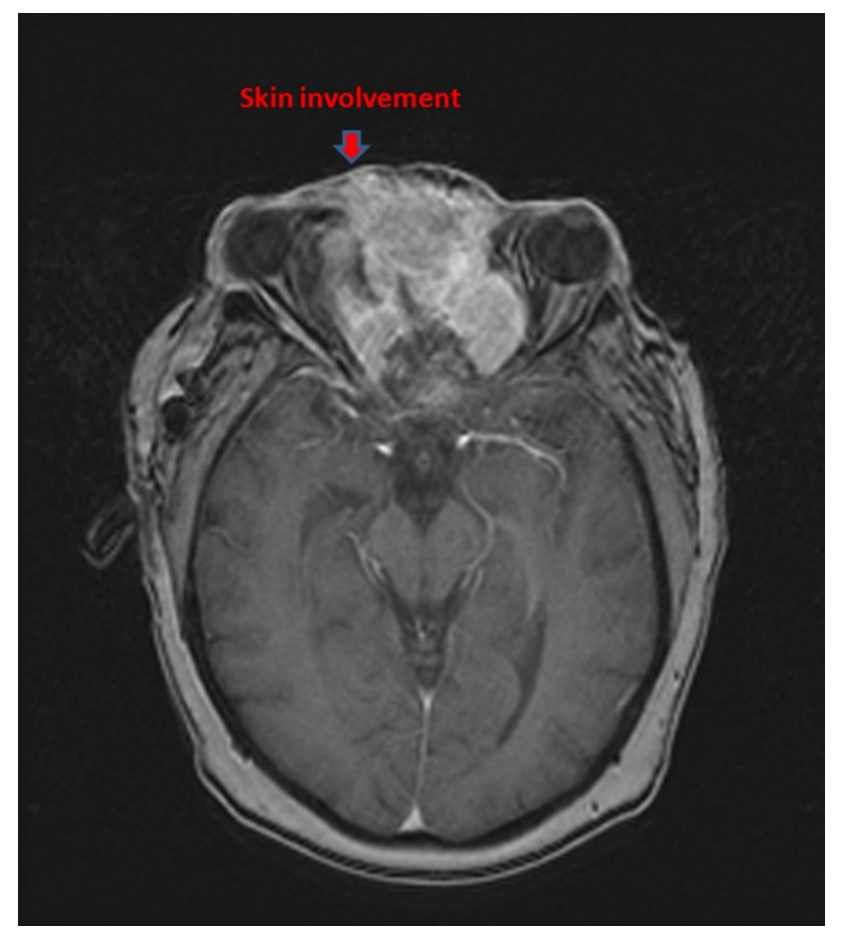

Figure 1. MRI of a patient with an unresectable esthesioneuroblastoma who presented with proptosis, lateral deviation of the left eye.

\section{Statistical analyses}

The primary endpoints for this study were relapse-free survival and overall survival. Survival statistics was calculated according to Kaplan-Meier from the date of primary therapy, whereas the disease free survival was calculated from the last day of the given treatment. Survival between groups was compared using the log-rank test. All data analyses were performed by IBM SPSS software (Ver. 23.0; Chicago, IL), with a $\mathrm{P}$ value of $<0.05$ considered statistically significant.

\section{Results}

\section{Patients' characteristics}

All 54 patients were employed for investigation of clinical characteristics of ENB. The age of onset of ENB revealed a bimodal distribution, with peaks at 10 20 and 50 60 years at primary diagnosis. Among those included, characteristics and treatment modalities were summarized in Table 1.

Among all included patients, nine patients were treated with surgery alone, 10 with surgery followed by radiotherapy, 1 with chemotherapy followed by surgery. Briefly, open craniofacial resection was performed in nine patients, with endoscopic-assisted craniofacial resection and endoscopic-definitive surgery in three patients each.

\section{Radiotherapy}

Six patients were treated with radiotherapy (RT) alone, 10 with surgery followed by RT, 15 with chemoradiotherapy. The technique for RT included 2D conventional RT in 25 patients, 3D conformal RT in five patients and intensity modulated RT (IMRT) in one patient. The median dose to the target volume was 63 Gy (range, 50-76 Gy), median dose per fraction was 2 Gy (range, 1.8 3Gy) with 5-10 MV photons of a linear accelerator.

\section{Chemotherapy}

Chemotherapy was delivered in 33 patients. Among those, 18 patients were treated with induction chemotherapy, three patients with chemotherapy after definitive radiotherapy/surgery and 15 with concurrent with definitive radiotherapy (3 of 15 received adjuvant chemotherapy simultaneously), and 1 with adjuvant chemotherapy. The regimens used for chemotherapy included CTX+ADM+VCR $(\mathrm{CAV}, \mathrm{n}=4), \mathrm{VP}-16+\mathrm{DDP}(\mathrm{EP}, \mathrm{n}=3), \mathrm{IFO}+\mathrm{ADM}+\mathrm{DTIC}$ (MAID, n=2), VCR+IFO+DDP (VIP, n=2), $\mathrm{VM} 26+\mathrm{IFO}+\mathrm{DDP} \quad(\mathrm{n}=2), \mathrm{CTX}+\mathrm{ADM}+\mathrm{DDP} \quad(\mathrm{CAP}$, $\mathrm{n}=1)$, and $\mathrm{CTX}+\mathrm{VCR}+\mathrm{ADM}+\mathrm{PDN}(\mathrm{CHOP}, \mathrm{n}=1)$, with a median of three cycles per patient (range,2-6 cycles) administered.

\section{Long-term Survival Investigation}

The median follow-up for this cohort was 84 months (range, 27-198 months). The 5-year overall and progression free survival rates were $42.7 \%$ and $39.1 \%$, 
respectively, with 10 -year rates of $28.9 \%$ and $21.7 \%$ respectively. Of note, 21 patients developed recurrent disease, and the 5, 10-year recurrent free survival rates were $51.7 \%$ and $34.0 \%$, respectively (Fig.2).

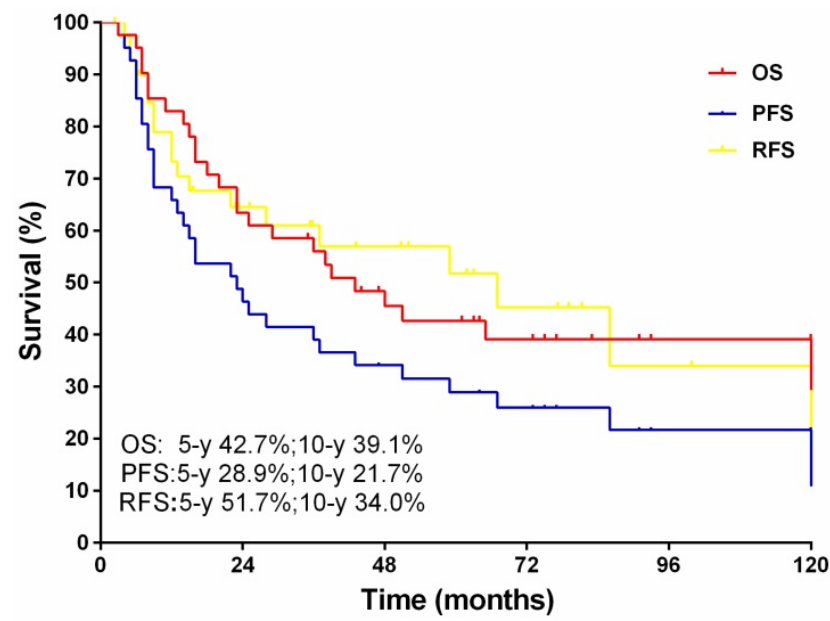

Figure 2. The overall survival, progression free survival and recurrence free survival.

\section{Survival in association with TNM staging}

The 5, 10-years overall survival rates according to AJCC T-category were $83.3 \%, 55.6 \%$ for $\mathrm{T} 1,46.7 \%, 46.7 \%$ for $\mathrm{T} 2,34.2 \%, 34.2 \%$ for $\mathrm{T} 3$, and $15.4 \%$, $15.4 \%$ for $\mathrm{T} 4$ respectively (Fig.3A). The 5, 10-years progression-free survival rates was $50 \%, 50 \%$ for $\mathrm{T} 1,37.3 \%, 14.0 \%$ for $\mathrm{T} 2,15.3 \%, 15.3 \%$ for $\mathrm{T} 3$, and
15.0\%, 0\%for T4 (Fig.3B). The 5,10-years overall survival rates according to N-category was $40.8 \%$, $35.7 \%$ for N0, and $44.4 \%, 44.4 \%$ for N1(Fig.3C). The 5, 10 - years progression-free survival rates were $27.2 \%$, $20.4 \%$ for N0, and $33.3 \%, 16.7 \%$ for N1 (Fig.3D). The overall survival and progress-free survival did not show significant correlation with tumor $\mathrm{T}$ - or $\mathrm{N}$-staging.

\section{Survival in association with Kadish/Morita staging}

The 5, 10-years overall survival rates according to Kadish/Morita staging was $100 \%, 66.7 \%$ for stage A, $43.8 \%, 43.8 \%$ for stage B, $38.7 \%, 38.7 \%$ for stage C, and $28.6 \%, 28.6 \%$, for stage D (Fig.3E). The 5, 10- years progression-free survival rates were $66.7 \%, 66.7 \%$ for stage A, 30\%,15\% for stage B, 33.8\%,15.4\% for stage C, and $16.7 \%, 16.7 \%$ for stage D (Fig.3F). The difference between overall survival and progress-free survival also had no statistical significance on basis of the Kadish/Morita staging.

\section{Survival in association with skin involvement at initial diagnosis}

However, Patients with skin-involved ENB demonstrated significantly poorer 5-year overall survival $(0 \%$ vs.45.5\%, $\mathrm{P}=0.038)$ and 5-year progress-free survival ( $0 \%$ vs. $31.3 \%, P=0.001)$ than those without skin involvement (Fig.3G, I).
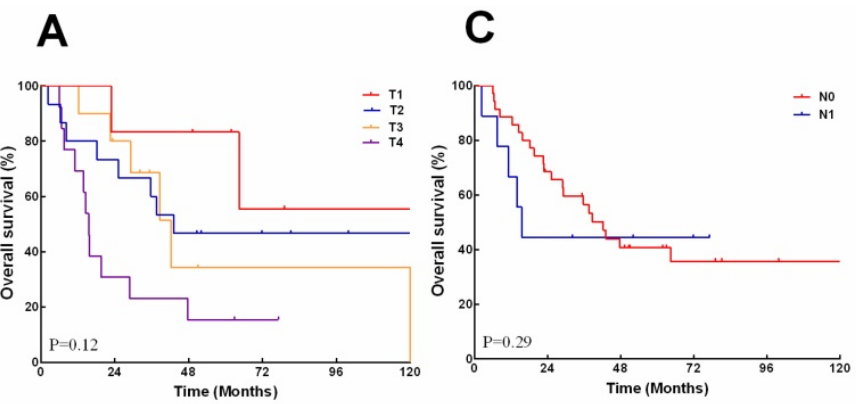

E
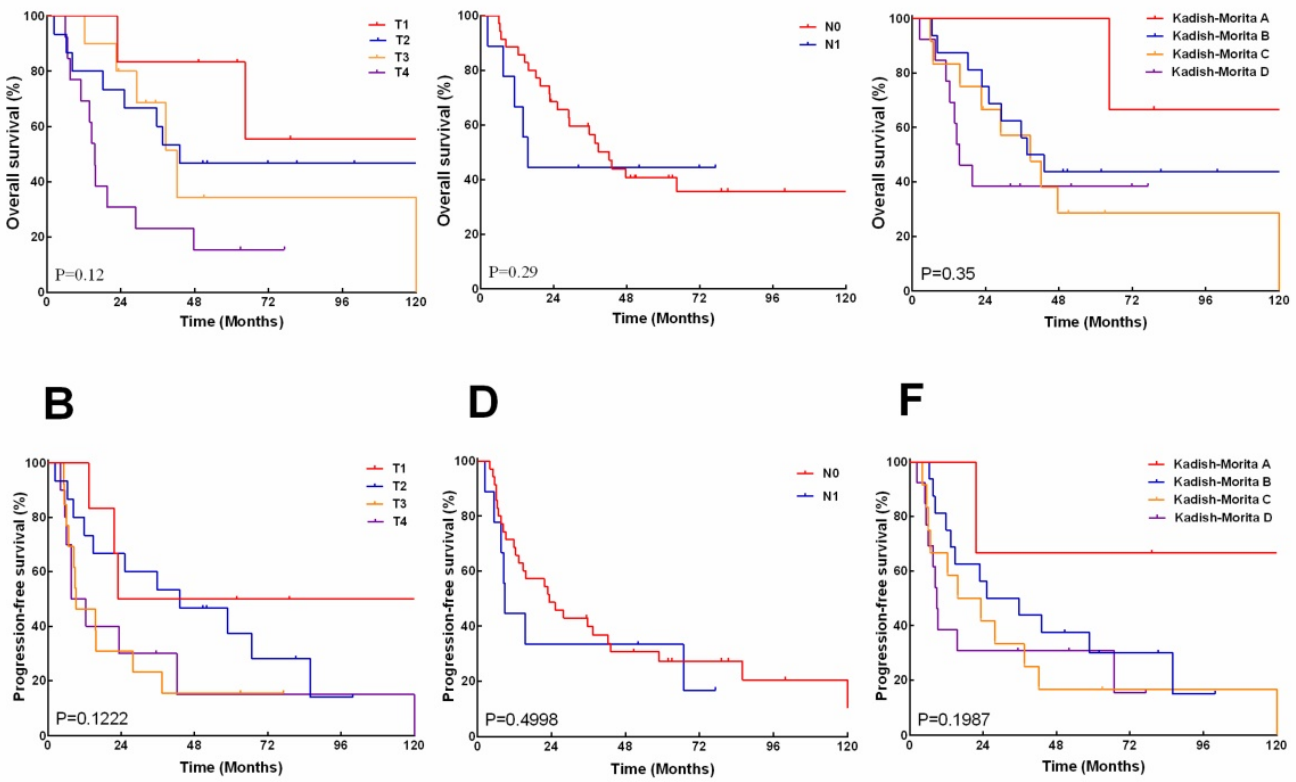

D

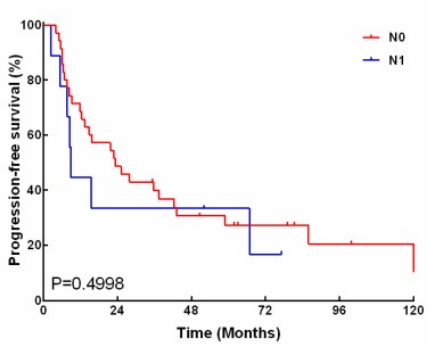

$\mathbf{F}$

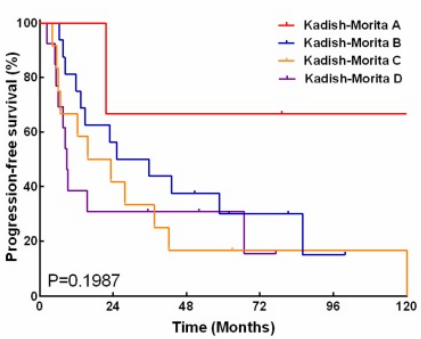

H

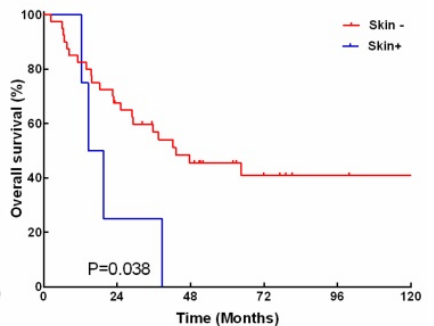

I

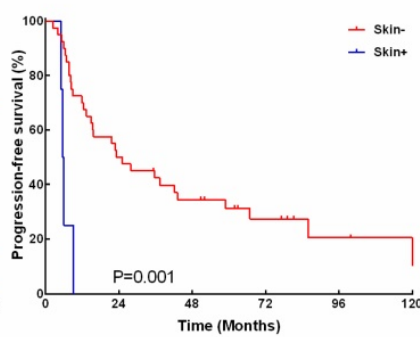

Figure 3. The overall survival (A, C, E, H) and progression -free survival (B, D, F, I) stratified by American Joint Committee on Cancer (AJCC) T and N classification, modified Kadish score, and local skin involved. 

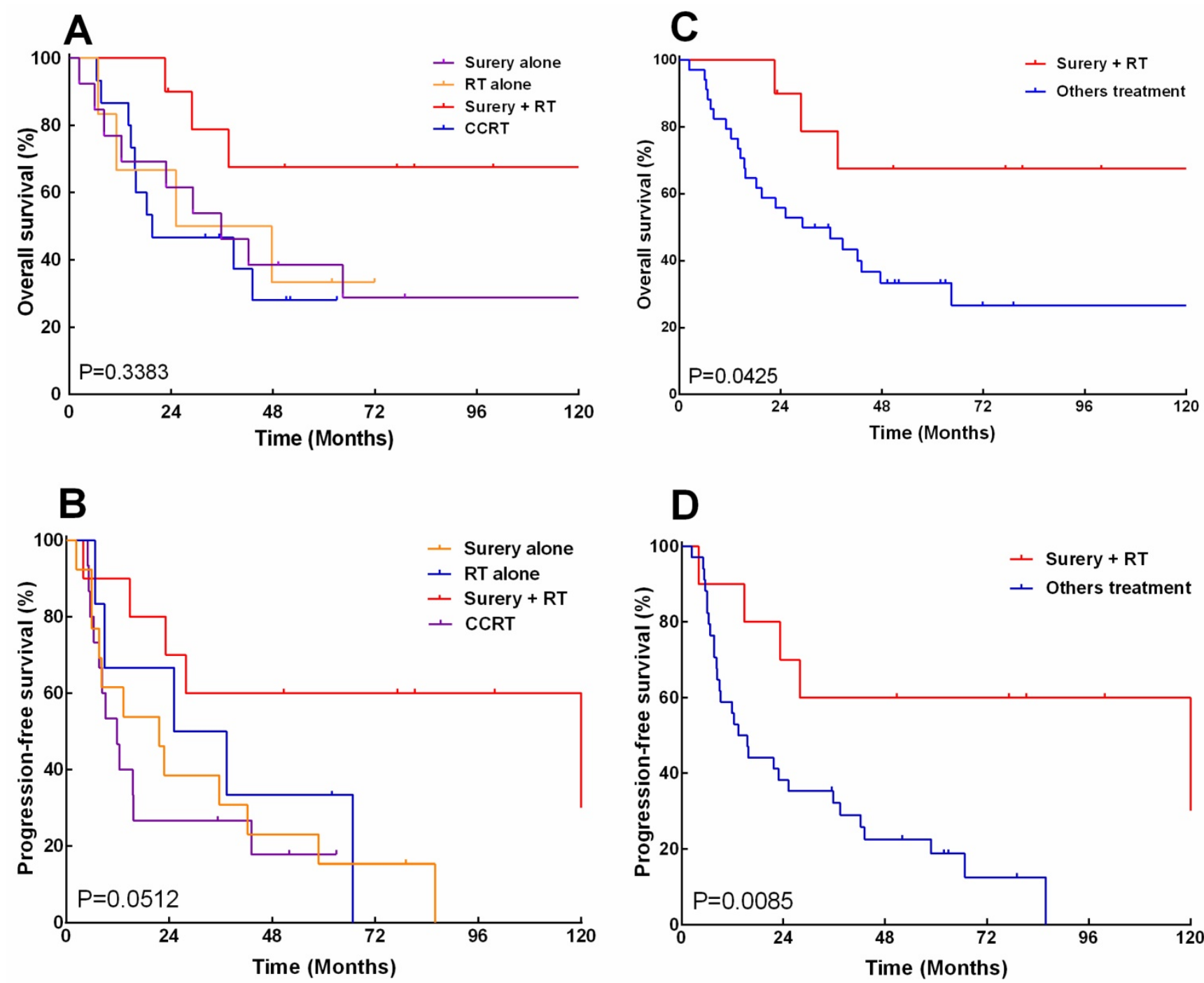

Figure 4. The overall survival $(\mathrm{A}, \mathrm{CH})$ and progression -free survival $(\mathrm{B}, \mathrm{D})$ of 44 patients with esthesioneuroblastoma (ENB) when stratified by treatment $(\mathrm{A})$ and stratified by those who received surgery and postoperative radiation versus other treatments.

\section{Survival in association with treatment modality}

The overall survival and progression-free survival were analyzed by log-rank test again, with patients stratified according to treatment modalities. The median overall survival for patients receiving surgery combined with RT was 138.6 months, as compared to 35.7, 36.4, and 19.6months for those undergoing surgery alone, radiation alone and CCRT, respectively. The 5-, 10-year overall survival rates were $67.5 \%$ and $67.5 \%$ for surgery combined with radiotherapy, as compared to $38.5 \%$ and $28.8 \%, 33.3 \%$ and $33.3 \%, 28 \%$ and $28 \%$ for surgery alone, radiation alone and CCRT, respectively (Fig.4A). Besides, the median progression-free survival for patients receiving surgery combined with radiotherapy was 121.7 months, as compared to 21.7, 31.3, and 11.9 months for those undergoing surgery alone, radiation alone and CCRT, respectively. The 5-year, 10-year progression-free survival rates were $67.5 \%$ and $67.5 \%$ for surgery combined with radiotherapy, as compared to $15.4 \%$ and $0 \%, 33.3 \%$ and $0 \%, 17.8 \%$ and $0 \%$ for surgery alone, radiation alone and CCRT, respectively (Fig. 4B). Overall, patients who received surgery combined with radiotherapy had significantly improved5-year overall survival $(67.5 \%$ vs. $33.3 \%$, $\mathrm{P}=0.043)$ and progress-free survival $(60.0 \%$ vs. $18.7 \%$, $\mathrm{P}=0.008), \quad$ as compared with other treatment approaches (Fig. 4C.D).

\section{Patterns of failure after treatment}

Overall, 21 patients(47.7\%) developed relapses or disease progression, which was occurred at $4 \sim 121$ months (median, 9 months).The local relapse was observed as first recurrence in 12 patients $(27.3 \%)$, with regional relapse in six patients $(13.6 \%)$ and distant metastasis in 3 patients (6.8\%). The distant 
metastasis occurred in five patients, which involving the subcutaneous tissue in one patient, the bone in three patients and the breast in one patient. Patients suffering from regional relapses and distant metastasis were mainly treated with chemotherapy and palliative care, however, all of them were poorly controlled with such measures. The 5-year, 10-year recurrent free survival rates were $51.7 \%$ and $34.0 \%$, respectively. The median survival time was 20 months for patients with simple local relapses and 14 months for those with regional and/or distant metastasis were 14 months (Table 2).

Table 2. Patterns of failure for patients with Esthesioneuroblastoma

\begin{tabular}{ll}
\hline Patterns of failure & No. of patients $(\%)$ \\
\hline Recurrence & \\
Yes & $21(47.7 \%)$ \\
No & $23(52.3 \%)$ \\
Site of first recurrence & \\
Local relapse & $12(27.3 \%)$ \\
Regional relapse & $6(13.6 \%)$ \\
Distant metastasis & $3(6.8 \%)$ \\
Site of distant metastasis & \\
Bone & $2(4.9 \%)$ \\
Breast and bone & $1(2.3 \%)$ \\
Involvement on frontal region & $1(2.3 \%)$ \\
\hline
\end{tabular}

Among 22 patients (50.0\%) with T1 or T2 disease, seven patients $(31.8 \%)$ developed local relapses, with only one regional relapse case without distant metastases. Within other 22 patients $(50.0 \%)$ with T3 or T4 disease, six patients $(27.3 \%)$ developed local relapses, with four regional relapses cases $(18.2 \%)$ and one case for regional and distant metastases recorded. Among patients with locoregional LN metastasis, two out of nine patients had local relapses, with one case for regional relapses, one case for regional nodal relapses and bone metastases, another one case for breast and bone metastases.

Among 19 patients with Kadish/Morita stage A or B disease, seven patients had local relapses (36.8\%), with recurrent local relapses after surgical salvage recorded in five cases. Four of those recurrent relapses abandoned further treatments. Of six cases receiving radiotherapy alone, one had merely a 4-month remission and one received only palliative care. Of 25 patients with stage or $\mathrm{D}$ disease, seven had local relapses $(28 \%)$ and eight had regional and distant metastases (32\%), with one case having local, regional and distant relapses simultaneously. Of seven patients with local relapses, two patients received surgical salvage but died from tumor progression.

Among four patients with skin involvement, the median survival time was 22 months. All those patients presented with local relapses $(100 \%)$, of whom two cases demonstrated a regional relapse. Although salvage chemotherapy or surgery was administered, all of them died from tumor progression.

\section{Discussion}

Our research has shown that ENB manifested with a bimodal distribution of age onset. Surgery combined with radiotherapy showed significantly improved 5-year overall survival and progress-free survival compared with other therapeutic approaches. ENB without skin involvement showed significantly better 5-year overall survival and progress-free survival than that with skin involvement. Locoregional recurrence was the main cause of treatment failure.

The diagnosis and treatment of ENB have improved significantly over the last decades. Our results have demonstrated that 5-year and 10-year overall survivals are superior in patients treated with surgery followed by postoperative radiotherapy to those treated with surgery or radiotherapy alone, which is consistent with recent reports that the combination of surgery and post-operational radiation in the craniofacial regions demonstrates better treatment outcome for ENB patients with 5 -year overall survival rates of $60-88 \% 17-19$. Gruber et al have reported that the efficacy of RT alone with higher than 73 Gy or combined with chemotherapy was poorer than surgery plus RT, especially for patients having been treated with radical resection ${ }^{20}$, 21. Due to its special location that ENB readily spreads from its original nasal cavity to peripheral structures at initial diagnosis, the extension of the resection have been expanded as much as possible to ensure complete or near complete tumor excision. Thus, the current therapeutic protocols for ENB consists mainly of surgical resection combined with radiotherapy, radiotherapy and/or chemotherapy could not be used to replace surgery.

The 5-year overall survival rate in our study was $42.7 \%$, which was much lower than that reported in the literature. Contributing factors may include: (1) A fairly large proportion of patients (30\%) with Kadish/Morita stage or B disease only received non-surgical treatments; (2) Most patients were treated with surgical procedures of lateral nasal incision, which might limit the range of surgical excision.

According to the modified Kadish/Morita staging system, stage A and B diseases are classified as a relatively limited tumor location, whereas stage $C$ and $\mathrm{D}$ diseases as more widespread tumor locations. Patients with stage A or B disease mainly received surgical resection combined with radiotherapy, 
whereas patients with stage $\mathrm{C}$ or $\mathrm{D}$ disease mainly received radiotherapy combined with non-surgical treatments. Since the ENB is a very invasive tumor and its diagnosis is often depended upon wide extension of the lesion, single treatment modality may not be as sufficient as combined patterns. Especially for patients with stage $\mathrm{C}$ or $\mathrm{D}$ disease, the tumor usually has invaded into the maxillary sinus from the nasal cavity or with neck LN metastasis, it seems difficult to treat with primary surgery or radiotherapy alone as the first-line treatment. Therefore, we consider that surgery followed by post-operational radiotherapy would be the optimal therapeutic protocol for ENB.

Due to its low incidence, the optimal treatment remains unclear. Whereas the benefit of chemotherapy has been described in the literature 22,23 . The chemotherapy protocols and specific chemotherapeutic drugs selected to treat patients with ENB may vary for each individual patient; it is difficult to analyze and compare their therapeutic effects. Studies have shown that effective chemotherapeutic drugs for ENB include DDP, VP-16, CTP-11, DOC; the CR rate for first-line chemotherapy was only $12-18 \%^{24-26}$. Newer chemotherapeutic drugs are constantly discovered, providing more opportunities to improve the efficacy of chemotherapy for ENB. Therefore, further clinical studies are needed to evaluate therapeutic benefits of chemotherapeutic protocols and specific drugs for ENB.

As for patients with other types of head and neck cancer, radiotherapy plays an important role in treatment or prophylactic therapy of neck LN metastasis. Monroe et al has showed that 11 patients receiving prophylactic neck radiation had no relapses, whereas nine patients without precautionary irradiation experienced a relapse rate of $44 \% 27$. In our study, nine patients with LN metastasis who received neck irradiation had no local relapse in the field of radiation, although one patient experienced recurrence at the contra lateral neck without irradiation. Our findings also indicate that radiotherapy would be a valuable treatment of occult metastasis in the neck LN from ENB. Among 33 patients without LN metastasis who did not receive precautionary neck irradiation, five patients experienced neck relapses(15.2\%),especially for stage $\mathrm{C}$ disease, the relapse rate in the neck was25\% (4/16 patients). Besides, $80 \%$ of patients with $\mathrm{LN}$ relapses were found in the upper neck including IB, II, and III areas. According to these observations we consider that patients in stage D disease with LN metastasis require only irradiation to the neck $\mathrm{LN}$ instead of neck dissection.
There are several limitations that should be mentioned. It is a retrospective analysis over a long period; while the low incidence of ENB limits the ability to determine the optimal treatment strategies in this study. Multi-center clinical trial is indispensable for further investigation to improve the treatment efficacy. The association of Hyams grading system with prognostic outcomes should be assessed28; however, this information was not available for the evaluation of the majority of clinicopathologic characteristics in this study.

In summary, patients with ENB could achieve favorable long-term survival when treated with surgical resection followed by postoperative radiotherapy, even though the lesion is locally advanced. Long-term observation is warranted since locoregional recurrence and mortality remains high. Multi-center clinical trial on improving treatment efficacy is indispensable for further investigation.

\section{Acknowledgements}

This research was supported by:

1. Medical science and technology research fund project in Guangdong province: A2015112;

2. National Natural Science Foundation of China (No. 81401307).

\section{Author contributions}

Conceived and designed the research: BW, XC. Performed the researches: YR, YY and CW. Analyzed the data: TZ, YY, and YH. Contributed reagents/materials/analysis tools: $\mathrm{YR}, \mathrm{YY}$, and TZ, JD Wrote the manuscript: YR. Edited and revised manuscript: WX.

\section{Competing Interests}

The authors have declared that no competing interest exists.

\section{References}

1 Hollen TR, Morris CG, Kirwan JM, et al. Esthesioneuroblastoma of the nasal cavity. Am J Clin Oncol. 2015; 38:311-314.

2 Jouini R, Ben BE, Ben GO, et al. [A rare tumor of the nasal cavity: esthesioneuroblastoma]. Tunis Med. 2011; 89:718.

3 Oskouian RJ, Jane JS, Dumont AS, Sheehan JM, Laurent JJ, Levine PA. Esthesioneuroblastoma: clinical presentation, radiological, and pathological features, treatment, review of the literature, and the University of Virginia experience. Neurosurg Focus. 2002; 12:e4.

4 Matsunaga M, Nakagawa T, Sakamoto $T$, Ito J. Sphenoid esthesioneuroblastoma arising from the hindmost olfactory filament. Auris Nasus Larynx. 2015; 42:170-172.

5 Kumar A, Sethi B, Kumar Y, Mishra JP. Esthesioneuroblastoma arising from the middle meatus. J Cancer Res Ther. 2013; 9:99-101.

6 Dulguerov P, Allal AS, Calcaterra TC. Esthesioneuroblastoma: a meta-analysis and review. Lancet Oncol. 2001; 2:683-690.

7 Eriksen JG, Bastholt L, Krogdahl AS, Hansen O, Joergensen KE. Esthesioneuroblastoma--what is the optimal treatment? Acta Oncol. 2000; 39:231-235.

8 Schwartz JS, Palmer JN, Adappa ND. Contemporary management of esthesioneuroblastoma. Curr Opin Otolaryngol Head Neck Surg. 2016; 24:63-69.

9 Giridhar P, Mallick S, Laviraj MA, Bhasker S. Esthesioneuroblastoma with large intracranial extension treated with Induction chemotherapy, de-bulking 
surgery and image guided intensity modulated radiotherapy. Eur Arch Otorhinolaryngol. 2015-06-04.

10 Kumar R. Esthesioneuroblastoma: Multimodal management and review of literature. World I Clin Cases. 2015; 3:774-778.

11 Papacharalampous GX, Vlastarakos PV, Chrysovergis A, Saravakos PK, Kotsis GP, Davilis DI. Olfactory neuroblastoma (esthesioneuroblastoma): towards minimally invasive surgery and multi-modality treatment strategies - an updated critical review of the current literature. J BUON. 2013; 18:557-563.

12 Yin ZZ, Luo JW, Gao L, et al. Spread patterns of lymph nodes and the value of elective neck irradiation for esthesioneuroblastoma. Radiother Oncol. 2015; 117:328-332

13 Van Gompel JJ, Link MJ, Sheehan JP, et al. Radiosurgery is an Effective Treatment for Recurrent Esthesioneuroblastoma: A Multicenter Study. J Neurol Surg B Skull Base. 2014; 75:409-414.

14 Noh OK, Lee SW, Yoon SM, et al. Radiotherapy for esthesioneuroblastoma: is elective nodal irradiation warranted in the multimodality treatment approach? Int I Radiat Oncol Biol Phys. 2011; 79:443-449.

15 Mohindra S, Dhingra S, Mohindra S, Kumar N, Gupta B. Esthesioneuroblastoma: Good Local Control of Disease by Endoscopic and Endoscope Assisted Approach. Is it Possible? Indian J Otolaryngol Head Neck Surg. 2014; 66:241-247.

16 Gallia GL, Reh DD, Salmasi V, Blitz AM, Koch W, Ishii M. Endonasal endoscopic resection of esthesioneuroblastoma: the Johns Hopkins Hospital experience and review of the literature. Neurosurg Rev. 2011; 34:465-475.

17 Tajudeen BA, Arshi A, Suh JD, et al. Esthesioneuroblastoma: an update on the UCLA experience, 2002-2013. J Neurol Surg B Skull Base. 2015; 76:43-49.

18 De Bonnecaze G, Lepage B, Rimmer J, et al. Long-term carcinologic results of advanced esthesioneuroblastoma: a systematic review. Eur Arch Otorhinolaryngol. 2016; 273:21-26

19 Ow TJ, Hanna EY, Roberts DB, et al. Optimization of long-term outcomes for patients with esthesioneuroblastoma. Head Neck.2014; 36:524-530.

20 Gruber G, Laedrach K, Baumert B, Caversaccio M, Raveh J, Greiner R. Esthesioneuroblastoma: irradiation alone and surgery alone are not enough. Int I Radiat Oncol Biol Phys.2002; 54:486-491.

21 Hwang SK, Paek SH, Kim DG, Jeon YK, Chi JG, Jung HW. Olfactory neuroblastomas: survival rate and prognostic factor. I Neurooncol. 2002; 59:217-226.

22 Sohrabi S, Drabick JJ, Crist H, Goldenberg D, Sheehan JM, Mackley HB. Neoadjuvant concurrent chemoradiation for advanced esthesioneuroblastoma: a case series and review of the literature. J Clin Oncol. 2011; 29:e358-e361.

23 Aljumaily RM, Nystrom JS, Wein RO. Neoadjuvant chemotherapy in the setting of locally advanced olfactory neuroblastoma with intracranial extension. Rare Tumors.2011; 3:e1.

24 Fury MG, Sherman E, Haque S, et al. A phase I study of daily everolimus plus low-dose weekly cisplatin for patients with advanced solid tumors. Cancer Chemother Pharmacol. 2012; 69:591-598.

25 Kiyota N, Tahara M, Fujii S, et al. Nonplatinum-based chemotherapy with irinotecan plus docetaxel for advanced or metastatic olfactory neuroblastoma: a retrospective analysis of 12 cases. Cancer. 2008; 112:885-891.

26 Kim HJ, Kim $\mathrm{CH}$, Lee BJ, et al. Surgical treatment versus concurrent chemoradiotherapy as an initial treatment modality in advanced olfactory neuroblastoma. Auris Nasus Larynx. 2007; 34:493-498.

27 Monroe AT, Hinerman RW, Amdur RJ, Morris CG, Mendenhall WM. Radiation therapy for esthesioneuroblastoma: rationale for elective neck irradiation. Head Neck. 2003; 25:529-534.

28 Bell D, Saade R, Roberts D, et al. Prognostic Utility of Hyams Histological Grading and Kadish-Morita Staging Systems for Esthesioneuroblastoma Outcomes. Head Neck Pathol. 2015; 9:51-59 